\title{
Comparative Study between Fuzzified Newton Raphson Method and Original Newton Raphson Method and its Computer Application
}

\author{
Toralima Bora \\ Assistant Professor \\ Centre for Computer Studies \\ Dibrugarh University
}

\author{
G. C. Hazarika \\ Professor \\ Department of Mathematics \\ Dibrugarh University
}

\begin{abstract}
Numerical methods are techniques which give approximate but accurate solutions to difficult class of problems.

Fuzzy numbers are foundation of fuzzy sets and fuzzy mathematics that extend the domain of numbers from those of real numbers to fuzzy numbers.

Researchers in the past investigated a number of methods of numerical analysis with the help of Fuzzy theory. Recently, various methods have been developed for solving linear programming problems with fuzzy number. Many research works have been done on fuzzy numbers and on its applications in various fields. But very few developments have been seen in the area of numerical methods using fuzzy triangular numbers and actual computer codes.

In this paper the fuzzification of Newton Raphson method to find the solution of cubic equation has been discussed. Results have been obtained in the form of triangular numbers along with the membership functions using computer programs. The root obtained is then defuzzified using centroid method to convert it into crisp number. Also computer codes are developed for the Newton Raphson Method. Finally comparison has been made between the results obtained from the two methods.
\end{abstract}

\section{Keywords}

Newton Raphson Method, Fuzzy membership function, Triangular fuzzy number, $\alpha$-cut etc.

\section{INTRODUCTION}

Numerical methods are techniques which give approximate but accurate solutions to difficult class of problems.

Wang Y. [2014] in his paper entitled "On Theory of Fuzzy Numbers and Fuzzy Arithmetic" explained that Fuzzy numbers in number theory are foundation of fuzzy sets and fuzzy mathematics that extend the domain of numbers from those of real numbers to fuzzy numbers. Fuzzy arithmetic is a system of fuzzy operations on fuzzy numbers.

Researchers in the past investigated a number of methods of numerical analysis with the help of Fuzzy theory. Recently, Zhong Y. et al. [2013] in their research paper discussed that various methods have been developed for solving linear programming problems with fuzzy number, such as simplex method and dual simplex method. But their computational complexities are exponential, which is not satisfactory for solving large scale fuzzy linear programming problems, especially in the engineering field. In some research work an alternative method was proposed for solving fuzzy number linear programming problems. Many research works were done on fuzzy numbers and on its applications in various fields. But very few developments have been seen in the area of numerical methods using fuzzy triangular numbers.

The uncertainty associated with numerical methods can be tackled by using fuzzy logic. The study relating to Fuzzification of Mathematical Methods has not been undertaken much to find the fuzzified general formula of the Numerical Methods. Taking these as research gap, effort will be made in this study to fill this gap to some extent and to add to the existing stock of knowledge. Therefore in this study the topic of Fuzzification will be studied with reference to Newton Raphson Method . Apart from studying the fuzzification practice of Newton Raphson Method, effort will also be made to study its computer application. The root of the cubic equation which will be obtained from the Fuzzified Newton Raphson Method, will then be defuzzified using Centroid Method to get the equivalent crisp number. Finally comparison will be made between the results obtained from the two methods.

\section{OBJECTIVE OF THE STUDY}

The main objective of the study is to fuzzify Newton Raphson Method and to develop computer program for the same. The specific objectives are

- Fuzzification of Newton-Raphson Method and to develop computer program for the same.

- Defuzzification of the triangular number obtained from the Fuzzified Newton Raphson Method.

- Compare the outputs of Fuzzified Newton Raphson Method and original Newton Raphson Method.

\section{METHODOLOGY}

Fuzzification of the Newton Raphson method will be done using triangular fuzzy number. Centroid Method will be used for the defuzzification of the triangular number to find the equivalent crisp number.

\section{FUZZIFICATION OF NEWTON RAPHSON METHOD \\ Let us consider $\mathrm{F}(\mathrm{X})=0$}

Let the function $\mathrm{F}(\mathrm{X})$ changes its sign over an interval $\mathrm{X}=\mathrm{A}$ and $\mathrm{X}=\mathrm{B}$.

Let $A=\left[A_{1}, A_{2}, A_{3}\right]$ and $B=\left[B_{1}, B_{2}, B_{3}\right]$. Then there is a root of $F(X)=0$ lying between $A$ and $B$. Now fuzzy membership function of $\mathrm{A}$ and $\mathrm{B}$ are respectively, 


$$
\begin{gathered}
\mu_{A}(X)=\left\{\begin{array}{l}
\frac{X-A_{1}}{A_{2}-A_{1}}, A_{1} \leq X \leq A_{2} \\
\frac{X-A_{3}}{A_{2}-A_{3}}, A_{2} \leq X \leq A_{3} \\
0, \text { otherwise }
\end{array}\right. \\
\mu_{B}(X)=\left\{\begin{array}{l}
\frac{X-B_{1}}{A_{2}-A_{1}}, B_{1} \leq X \leq B_{2} \\
\frac{X-B_{3}}{B_{2}-B_{3}}, B_{2} \leq X \leq B_{3} \\
0, \text { otherwise }
\end{array}\right.
\end{gathered}
$$

with respect to $\alpha$-cuts as

$$
\begin{aligned}
& {[A]^{\alpha}=\left[A_{1}+\alpha\left(A_{2}-A_{1}\right), A_{3}+\alpha\left(A_{2}-A_{3}\right)\right]} \\
& {[B]^{\alpha}=\left[B_{1}+\alpha\left(B_{2}-B_{1}\right), B_{3}+\alpha\left(B_{2}-B_{3}\right)\right]}
\end{aligned}
$$

As a first approximation, the root of $\mathrm{F}(\mathrm{X})=0$ is $X_{0}=A$ or $B$ Let $X_{0}=A=\left[A_{1}, A_{2}, A_{3}\right]$

$$
\mu_{X_{0}}(X)=\left\{\begin{array}{l}
\frac{X-A_{1}}{A_{2}-A_{1}}, A_{1} \leq X \leq A_{2} \\
\frac{X-A_{3}}{A_{2}-A_{3}}, A_{2} \leq X \leq A_{3} \\
0, \text { otherwise }
\end{array}\right.
$$$$
\left[X_{0}\right]^{\alpha}=\left[A_{1}+\alpha\left(A_{2}-A_{1}\right), A_{3}+\alpha\left(A_{2}-A_{3}\right)\right]
$$

According to Newton Raphson formula

$$
X_{1}=X_{0}-\frac{F\left(X_{0}\right)}{F^{\prime}\left(X_{0}\right)}=\left[X_{1}^{\prime}, X_{1}^{\prime \prime}, X_{1}^{\prime \prime \prime}\right]
$$

with membership function

$$
\mu_{X_{1}}(X)=\left\{\begin{array}{c}
\frac{X-X_{1}^{\prime}}{X_{1}^{\prime \prime}-X_{1}^{\prime}}, X_{1}^{\prime} \leq X \leq X_{1}^{\prime \prime} \\
\frac{X-X_{1}^{\prime \prime}}{X_{1}^{\prime \prime}-X_{1}^{\prime \prime}}, X_{1}^{\prime \prime} \leq X \leq X_{1}^{\prime \prime} \\
0, \text { otherwise }
\end{array}\right.
$$

and $\left[X_{1}\right]^{\alpha}=\left[X_{1}^{\prime}+\alpha\left(X_{1}^{\prime \prime}-X_{1}^{\prime}\right), X_{1}^{\prime \prime \prime}+\alpha\left(X_{1}^{\prime \prime}-X_{1}^{\prime \prime \prime}\right)\right]$ similarly

$$
X_{2}=X_{1}-\frac{F\left(X_{1}\right)}{F,\left(X_{1}\right)}=\left[X_{2}^{\prime}, X_{2}^{\prime \prime}, X_{2}^{\prime \prime \prime}\right]
$$

with membership function

$$
\mu_{X_{2}}(X)=\left\{\begin{array}{c}
\frac{X-X_{2}^{\prime}}{X_{2}^{\prime \prime}-X_{2}^{\prime}}, X_{2}^{\prime} \leq X \leq X_{2}^{\prime \prime} \\
\frac{X-X_{2}^{\prime \prime}}{X_{2}^{\prime \prime}-X_{2}^{\prime \prime}}, X_{2}^{\prime \prime} \leq X \leq X_{2}^{\prime \prime \prime} \\
0, \text { otherwise }
\end{array}\right.
$$

and $\left[X_{2}\right]^{\alpha}=\left[X_{2}^{\prime}+\alpha\left(X_{2}^{\prime \prime}-X_{2}^{\prime}\right), X_{2}^{\prime \prime \prime}+\alpha\left(X_{2}^{\prime \prime}-X_{2}^{\prime \prime \prime}\right)\right]$

Again

$X_{3}=X_{2}-\frac{F\left(X_{2}\right)}{F^{\prime}\left(X_{2}\right)}=\left[X_{3}^{\prime}, X_{3}^{\prime \prime}, X_{3}^{\prime \prime \prime}\right]$

with membership function

$$
\mu_{X_{3}}(X)=\left\{\begin{array}{l}
\frac{X-X_{3}^{\prime}}{X_{3}^{\prime \prime}-X_{3}^{\prime}}, X_{3}^{\prime} \leq X \leq X_{3}^{\prime \prime} \\
\frac{X-X_{3}^{\prime \prime}}{X_{3}^{\prime \prime}-X_{3}^{\prime \prime}}, X_{3}^{\prime \prime} \leq X \leq X_{3}^{\prime \prime} \\
0, \text { otherwise }
\end{array}\right.
$$

and $\left[X_{3}\right]^{\alpha}=\left[X_{3}^{\prime}+\alpha\left(X_{3}^{\prime \prime}-X_{3}^{\prime}\right), X_{3}^{\prime \prime \prime}+\alpha\left(X_{3}^{\prime \prime}-X_{3}^{\prime \prime \prime}\right)\right]$
Similarly

$$
X_{4}=X_{3}-\frac{F\left(X_{3}\right)}{F,\left(X_{3}\right)}=\left[X_{4}^{\prime}, X_{4}^{\prime \prime}, X_{4}^{\prime \prime \prime}\right]
$$

with membership function

$$
\mu_{X_{4}}(X)=\left\{\begin{array}{l}
\frac{X-X_{4}^{\prime}}{X_{4}^{\prime \prime}-X_{4}^{\prime}}, X_{4}^{\prime} \leq X \leq X_{4}^{\prime \prime} \\
\frac{X-X_{1}^{\prime \prime}}{X_{4}^{\prime \prime}-X_{4}^{\prime \prime}}, X_{4}^{\prime \prime} \leq X \leq X_{4}^{\prime \prime \prime} \\
0, \text { otherwise }
\end{array}\right.
$$

and $\left[X_{4}\right]^{\alpha}=\left[X_{4}^{\prime}+\alpha\left(X_{4}^{\prime \prime}-X_{4}^{\prime}\right), X_{4}^{\prime \prime \prime}+\alpha\left(X_{4}^{\prime \prime}-X_{4}^{\prime \prime \prime}\right)\right] \quad$ and so on.

\section{COMPUTER APPLICATION OF FUZZIFIED NEWTON RAPHSON METHOD}

Let us consider a cubic equation

$$
X^{3}-4 X-9=0
$$

Let the root lies between two fuzzy triangular numbers $A=[-.01,0, .01]$ and $B=[.99,1,1.01]$

Since

$$
F(A)=F([-.01,0, .01])=A^{3}-6 A+4
$$

\begin{tabular}{|c|c|c|}
\hline Iteration & $\begin{array}{lc}\text { Root } \quad x & \text { (fuzzy } \\
\text { triangular no) } & \\
\end{array}$ & $\begin{array}{l}\text { Defuzzified } \\
\text { Number }\end{array}$ \\
\hline 1 & 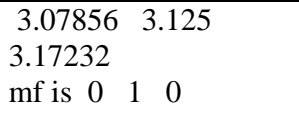 & 3.1253 \\
\hline 2 & 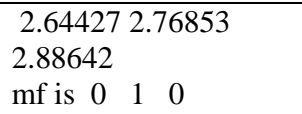 & 2.7664 \\
\hline 3 & 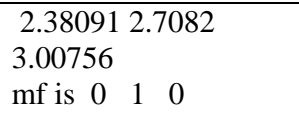 & 2.6989 \\
\hline 4 & 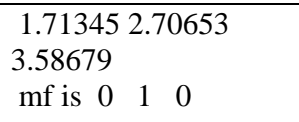 & 2.6689 \\
\hline
\end{tabular}

$=[3.93,4,4.07]$

$$
\begin{aligned}
& =+\mathrm{ve} \\
& F(B)=F([.99,1,1.01])=B^{3}-6 B+4
\end{aligned}
$$

$=[-1.09,-1,-.89]$

$$
=\text {-ve }
$$

Since $F(A)$ and $F(B)$ are of opposite sign, a root lies between $[-.01,0, .01]$ and $[.99,1,1.01]$.

Let us take $X_{0}=[-.99,1,1.01]$

Two computer programs have been developed to find the solution of the considered cubic equation.

The following table shows the output of the program developed for Fuzzified Newton Raphson Method.

Table 1 
The Table 1 shows the output of the program developed to find the solution of the said equation using Fuzzified Newton Raphson Method. It has been observed that the root is obtained after $4^{\text {th }}$ iteration in the form of triangular fuzzy number along with its membership function in Table 1 . The third column of Table 1 gives the defuzzified value of the corresponding Fuzzy Triangular Number given in the second column of the same table. Defuzzification is the conversion of one or more fuzzy sets into a number that can be said to represent the fuzzy sets. The Centroid Method is used to defuzzify the fuzzy triangular number. Therefore, a decision has been made on the defuzzified value obtained. In the above table the third column shows the defuzzified value of the corresponding fuzzy triangular number given in the second column.

The following table shows the output of the program developed for original Newton Raphson Method

Table 2

\begin{tabular}{|c|c|}
\hline Iteration & Root $\mathrm{x}$ \\
\hline 1 & 3.125000 \\
\hline 2 & 2.768530 \\
\hline 3 & 2.708196 \\
\hline 4 & 2.706529 \\
\hline 5 & 2.706528 \\
\hline 6 & 2.706528 \\
\hline
\end{tabular}

The Table 2 shows the output of the program developed to find the solution of the given equation using Newton Raphson Method. From the Table 2 it has been observed that the root is obtained in the $6^{\text {th }}$ iteration.

\section{CONCLUSION}

In this study it has been observed that the root obtained using both the Fuzzified Newton Raphson Method and original Newton Raphson Method are almost same. But the Fuzzified Newton Raphson Method gives the output in the $4^{\text {th }}$ iteration whereas the original Newton Raphson Method gives the output in the $6^{\text {th }}$ iteration. Finally, comparing the root obtained from both the methods conclusion has been drawn that the Fuzzified Newton Raphson method gives the root rapidly in comparison to the original Newton Raphson Method.

\section{REFERENCES}

[1] Allahviranloo T., Asari S., "Numerical Solution of Fuzzy Polynomials by Newton-Raphson Method" Journal of Applied Mathematics Islamic Azad University of Lahijan,, Vol.7, [2011],pp17-24.

[2] Allahviranloo. T., Otadi M. and Mosleh M., "Iterative method for fuzzy equations" Soft Computing, Vol. 12 (2007), pp.935-939.

[3] Akram S., Ann Q.U., "Newton Raphson Method", International Journal of Scientific \& Engineering Research, Volume 6, Issue 7, [2015] .

[4] Ehiwario, J.C., Aghamie, S.O. "Comparative Study of Bisection, Newton-Raphson and Secant Methods of RootFinding Problems", IOSR Journal of Engineering (IOSRJEN), Vol. 04, Issue 04 [ 2014].

[5] Kajani M.T., Asady B., and Vencheh A.H., "An iterative method for solving dual fuzzy nonlinear equation", Applied Mathematics and Computation Vol.167 (2005), pp.316-323.

[6] Solanki C., Thapliyal P., Tomar K., "Role of Bisection Method", International Journal of Computer Applications Technology and Research, Volume 3- Issue 8, 535 - 535, [2014].

[7] Seikh M. R., Nayak P.K. and Pal M., "Generalized Triangular Fuzzy Numbers In Intuitionistic Fuzzy Environment" , International Journal of Engineering Research and Development, Volume 5, Issue 1,[ 2012], pp. 08-13 8.

[8] Tanakan S,." A New Algorithm of Modified Bisection Method for on linear Equation", Applied Mathematical Sciences, Vol. 7, [2013], $6107-6114$.

[9] Wang Y., "On Theory of Fuzzy Numbers and Fuzzy Arithmetic", Advances in Applied and Pure Mathematics, [2014].

[10] Zhong Y., Jia Y., Chen D., and Yang Y., "Interior Point Method for Solving Fuzzy Number Linear Programming Problems Using Linear Ranking Function", Journal of Applied Mathematics ,[ 2013]. 